\title{
Cooperative estimation algorithms for multi- sensor networks with imprecise measurements
}

\author{
Jianxin Chen ${ }^{1}$, Xinzhuo Ren ${ }^{1}$, Yinfei Xu ${ }^{1}$, Haojie Meng ${ }^{1}$, Zhenfan $\mathrm{Zhao}^{2,{ }^{*}}$, and Jijun Tang ${ }^{3}$ \\ ${ }^{1}$ Hangzhou Electric Equipment Manufacturing Co., Ltd. Hangzhou, China \\ ${ }^{2}$ School of Automation, Hangzhou Dianzi University, Hangzhou, China \\ ${ }^{3}$ Zhejiang Dayou Industrial Co., Ltd.Hangzhou, China
}

\begin{abstract}
A cooperative estimation algorithm is proposed for mutli-sensor networks with imprecise measurements caused by electromagnetic interferences, abnormal currents and other faults in the multi-sensor measurement process. Adaptive schemes based on a reference model are introduced to overcome the adverse effects of multiplicative interference on the estimated information. Then, rigorous theoretical proofs are developed to analyze the adaptive estimation algorithm. Finally, numerical simulation results are carried out to verify the effectiveness of the theoretical analysis.
\end{abstract}

Keywords: Mutli-sensor network, Fault detection, Reference model, Adaptive estimation, Imprecise measurements.

\section{Introduction}

Due to the easy deployment, cost-effective, flexible, reliable and accurate characteristics, sensor networks are widely used [1-2], such as civil monitoring [18], indoor and outdoor environment monitoring [14-17], fire detection [19], traffic condition detection [20][25] and health monitoring [21]. A sensor network is consisted of a large number of intelligent sensor nodes. These nodes are dispersedly deployed in the monitoring area to cooperatively observe the specific target state. Sensor nodes are usually equipped with radio transceivers and microprocessors, allowing them to communicate with each other and calculate the collected data [3]. Thus, more and more scholars pay attention to the distributed collaborative estimation algorithms of multiple sensors in recent years.

Different from estimation algorithms with central processing nodes [22], there is no central processing node in distributed estimation algorithms [23]. The local estimation value of each sensor will enter the communication network and interact with neighbour sensors to achieve the consistency and stability of the estimation results. The pressure of multi-sensor system data processing is evenly distributed to each node, thereby enhancing the reliability and scalability of the system [4].

However, the high reliability of most distributed estimation algorithms is based on the absence of faults in the estimators. But in the actual condition, the sensors are always affected by the electromagnetic interferences, abnormal currents and other faults during operation,

\footnotetext{
*Corresponding author: 840534548@qq.com
} 
resulting in the accuracy of the estimate algorithm deviating from expectations. To solve this problem, some scholars have given different solutions from various perspectives. For example, for fault detection, a distributed sensor fault detection method was developed based on estimated residuals and adaptive thresholds [5], while some scholars defined the probability threshold for fault detection based on the estimation of the error covariance norm [10]. For fault estimation, the fault was estimated by calculating the Kullback-Leibler divergence between two probability density functions when the fault is covered by noise [6]. In addition, two finite frequency random indexes $h_{\infty}$ were utilized to measure the robustness of the estimation error, which helps to track the sensor fault parameters when studying the sensor fault problem in the control system. For fault-tolerant measurements, an improved Cubature-Kalman filtering method was proposed based on weighted average consistency for heterogeneous sensors [11], which can overcome the adverse effects caused by sensor failures. Furthermore, an adaptive neural network event trigger control scheme was constructed for nonlinear non-strict feedback multi-agent system with input saturations, unknown interferences and sensor failures. Although the proposed method aimed at control system, it still has great referential significance for the fault-tolerant measurements of multisensor systems. Some other scholars proposed a cluster-based fault-tolerant protocol [24], which utilized energy-saving technology to cluster and proposed a new error detection mechanism with high accuracy.

Note that most of the existing research results on cooperative estimations for multi-sensor networks rarely take the multiplicative fault into account, especially the fault that will lead to the reverse estimation direction. In this regard, this paper proposes an adaptive measurement method with a reference model based on the traditional multi-sensor distributed estimation algorithms, which can effectively overcome the adverse effects of multiplicative interference factor on the measurement results.

\section{Problem formulation}

We model the dynamics of the target to be measured as follows:

$$
\dot{s}(t)=A s(t)+B w(t), s(0)=s_{0}
$$

where $s(t) \in \mathbb{R}^{u}$ is the $u$-dimensional state of the system, $w(t) \in \mathbb{R}^{l}$ is the $l$-dimensional process noise or external disturbance, which is a time-invariant constant vector, $s_{0}$ is the initial state of the system, $A \in \mathbb{R}^{u \times u}$ is the time-invariant state matrix, $B \in \mathbb{R}^{u \times l}$ is the timeinvariant interference matrix.

Then, the measurement model of each sensor is given as:

$$
y_{i}(t)=C s(t)+D v_{i}(t)
$$

where $y_{i}(t) \in \mathbb{R}^{u}$ is the measurement output of the sensor $i, v_{i}(t) \in \mathbb{R}^{l}$ is the measurement or communication noise of the sensor, which is also a constant vector, $C \in \mathbb{R}^{u \times u}$ is the output measurement matrix, $D \in \mathbb{R}^{u \times l}$ is the output interference matrix.

\subsection{Multi-sensor system estimation model}

A communication topology diagram $G$ between multiple sensors is constructed firstly, the Laplace matrix $L$ is introduced to represent the interaction between sensors. Considering that the target system is monitored by n sensors. These sensors form a communication network that can be represented by a graph denoted as $G=(\vartheta, \varepsilon, \pi)$, where $\vartheta=\{1,2, \ldots, n\}$ is the set of these sensors, $\varepsilon=\{(i, j) \mid i, j \in \vartheta\}$ is a collection of edges with communication interactions, $\pi=\left[a_{i j}\right]_{n \times n}$ is a weighted adjacency matrix, if $(i, j) \in \varepsilon$, then $a_{i j}>0$, otherwise $a_{i j}=0$. 
Degree matrix of diagram $G$ is $\Delta=\operatorname{diag}\left\{d_{1}, d_{2}, \ldots, d_{n}\right\}, d_{i} \in\left\{d_{1}, d_{2}, \ldots, d_{n}\right\}$ is the number of sensors that communicating with the sensor $i$, the resulting Laplacian matrix of diagram $G$ is $L=\Delta-\pi$. Any sensor that resides within the communication range of the sensor $i$ is called a neighbor of the sensor $i$. Therefore, the neighbor set of the sensor can be expressed as $N_{i}=\left\{j \in v \mid p_{i j} \leq r_{i}\right\}$, where $p_{i j}$ is the distance between the sensors $i$ and $j$, and $r_{i}$ represents the radius of the sensor's measurement range. Therefore, the estimated model of each sensor can be expressed as:

$$
\left\{\begin{array}{c}
\dot{x}_{i}(t)=h_{i}(t) \\
h_{i}(t)=-\sum_{j \in N_{i}} a_{i j}\left(x_{i}(t)-x_{j}(t)\right)
\end{array}\right.
$$

where $x_{i}(t) \in \mathbb{R}^{u}$ is the estimated state of the sensor $i, N_{i}=\left\{j \in\{1, \ldots, n\} \backslash\{i\}: a_{i j} \neq 0\right\}$ is the set of neighbour nodes of the sensor $i, h_{i}(t)$ is the consensus error between the state of the estimated model in the sensor $i$ and its neighbouring sensors.

\subsection{Estimation model of multi-sensor system with multiplicative fault}

Due to the existence of faults such as electromagnetic interferences and abnormal currents, the accuracy of the estimation algorithm may be deviated from expectations, and even the consensus and stability of the collaborative estimation results cannot be achieved. In this article, two types of failures of the estimation model are considered. Then the estimation model can be modified by:

$$
\dot{x}_{i}(t)=b_{i} \tilde{h}_{i}(t)
$$

where $\tilde{h}_{i}(t) \in \mathbb{R}^{u}$ is the distributed collaborative estimation algorithm that needs to be designed, $b_{i} \in \mathbb{R}^{1}$ and $\varepsilon_{i} \in \mathbb{R}^{u}$ is interference factors caused by electromagnetic interferences, abnormal currents, external noises, etc. Note that $b_{i} \neq 0$ denotes a bounded multiplicative interference, representing an unknown estimated direction, while $b_{i}=1$ indicates that there is no multiplicative interference.

This article aims to design an adaptive algorithm with a reference model to overcome the influence of multiplicative interference factor $b_{i}$ on the system, so that the estimation result of each sensor can converge to the average of all sensor measurement values, that is, $\lim _{t \rightarrow \infty}\left(x_{i}(t)-\frac{1}{n} \sum_{j=1}^{n} y_{j}(t)\right)=0, \forall i, j=1, \ldots, n$.

Assumption 1. Both $y_{i}(t)$ and $\dot{y}_{i}(t)$ are bounded.

Remark 1. For simplicity, one-dimensional case is discussed in the following context, and all the results obtained in this paper can be established for $u>2$ by using Kronecker product.

\section{Algorithm design}

In multi-sensor system, the distributed collaborative estimation algorithm with unknown estimation direction is a problem to be solved. In this section, we will use a Nussbaum function to design an adaptive measurement algorithm with a reference model to solve this problem. First, we consider the following estimation model that only contains multiplicative interference factors:

$$
\dot{x}_{i}(t)=b_{i} \tilde{h}_{i}(t)
$$


where $\tilde{h}_{i}(t)$ is the estimation algorithm that needs to be designed to overcome the influence of the multiplicative interference factor $b_{i}$ on the system estimation results.

The reference model is defined as:

$$
\left\{\begin{array}{c}
\dot{z}_{i}(t)=h_{i}(t), z_{i}(0)=x_{i}(0) \\
h_{i}(t)=-\sum_{j \in N_{i}} a_{i j}\left(x_{i}(t)-x_{j}(t)\right)
\end{array}\right.
$$

where $z_{i}(t)$ represents the state of the reference model, and $h_{i}(t)$ represents the consensus error between the state of the estimated model in the sensor $i$ and in the neighboring sensor's.

Then the tracking error can be defined as:

$$
e_{i}(t)=x_{i}(t)-z_{i}(t)-y_{i}(t)
$$

Thus, the adaptive estimation algorithm is constructed as follows:

$$
\left\{\begin{array}{c}
\tilde{h}_{i}(t)=k_{i}^{2}(t) \cos \left(k_{i}(t)\right)\left(e_{i}(t)-h_{i}(t)-\dot{y}_{i}(t)\right) \\
\dot{k}_{i}(t)=e_{i}^{2}(t)-h_{i}(t) e_{i}(t)-e_{i}(t) \dot{y}_{i}(t)
\end{array}\right.
$$

where $k_{i}(t)$ is the adaptive item of $\tilde{h}_{i}(t), k_{i}^{2}(t) \cos \left(k_{i}(t)\right)$ is a smooth even function of Nussbaum type [7], $\dot{y}_{i}(t)$ represents the derivative of measurement output in the sensor $i$.

Remark 2. For the inaccurate measurement problem caused by the multiplicative interference factor, $\dot{y}_{i}(t)=0$ means that the measurement value of each sensor is timeinvariant, such as measuring a stationary target. By contrast, $\dot{y}_{i}(t) \neq 0$ means that the measurement value of each sensor is time-varying, such as measuring a dynamic target.

Lemma 1 [7]. $V(\cdot)$ and $k(\cdot)$ be the smooth function on the domain, $V(t) \geq 0, \forall t \in$ $\left[0, t_{f}\right), \mathbb{N}(\cdot)$ represents the smooth even function of Nussbaum-type, $\theta$ is a non-zero constant, if the following inequality can be established:

$$
V(t) \leq \int_{0}^{t}(\theta \mathbb{N}(k(\tau))+1) \dot{k}(\tau) d \tau+\text { const, } \forall t \in\left[0, t_{f}\right)
$$

where const is a suitable constant, then $V(t), k(t)$ and $\int_{0}^{t}(\theta \mathbb{N}(k(\tau))+1) \dot{k}(\tau) d \tau$ must be bounded on $\left[0, t_{f}\right)$.

Lemma 2 [9]. If $\mathcal{F}:[0, \infty) \rightarrow R$ is square-integrable, which is $\lim _{t \rightarrow \infty} \int_{0}^{t} \mathcal{F}^{2}(\tau) d \tau<\infty$. Then if $\dot{\mathcal{F}}(t), t \in[0, \infty)$ exists and is bounded, then $\lim _{t \rightarrow \infty} \mathcal{F}(t)=0$.

Lemma 3. For undirected connected graphs, the Laplacian matrix $L$ has an eigenvector $\mathrm{I}_{n}=[1,1, \ldots, 1]^{T}$ with zero eigenvalues and the corresponding elements are all 1 . The other eigenvalues of $L$ are all positive, let $\lambda_{n}(L) \geq \cdots \lambda_{2}(L) \geq \lambda_{1}(L)=0$ represent $n$ eigenvalues of $L$.

Lemma 4 [8]. Define a matrix Q of $(n-1) \times n$ as follows

$$
Q=\left(\begin{array}{ccccc}
-1+(n-1) \mu & 1-\mu & -\mu & \cdots & -v \\
-1+(n-1) \mu & -\mu & 1-\mu & \ddots & \vdots \\
\vdots & \vdots & \ddots & \ddots & -\mu \\
-1+(n-1) \mu & -\mu & \ldots & -\mu & 1-\mu
\end{array}\right)
$$

where $\mu=\frac{n-\sqrt{n}}{n(n-1)}$. Then $Q$ has the following properties,

$$
Q I_{n}=0_{n-1}, Q Q^{T}=E_{n-1}, Q^{T} Q=E_{n}-\frac{1}{n} I_{n} I_{n}^{T}
$$

where $\mathrm{E}_{n}$ is the identity matrix. For undirected connected graphs, the real parts of all eigenvalues of $Q L Q^{T}$ are positive numbers. 
Theorem 1. In an undirected and connected sensor network, if the measurement target is stationary and there holds $\sum_{i=1}^{n} z_{i}(0)=0$, then the cooperative estimation of multi-sensor system (5) can achieve average consensus under the adaptive estimation algorithm (8), that is, $\lim _{t \rightarrow \infty}\left(x_{i}(t)-\frac{1}{n} \sum_{j=1}^{n} y_{j}(t)\right)=0, \forall i, j=1, \ldots, n$.

Proof. Considering the Lyapunov function $V_{i}=\frac{1}{2} e_{i}^{2}$, derivative of $V_{i}$ at time $t$ can be obtained

$$
\begin{aligned}
\dot{V}_{i}= & e_{i} \dot{e}_{i} \\
= & e_{i}\left(\dot{x}_{i}-\dot{z}_{i}-\dot{y}_{i}\right) \\
= & e_{i}\left(b_{i} \tilde{h}_{i}-h_{i}-\dot{y}_{i}\right) \\
= & e_{i} b_{i} \tilde{h}_{i}-e_{i} h_{i}-e_{i} \dot{y}_{i} \\
= & e_{i} b_{i} k_{i}^{2} \cos \left(k_{i}\right)\left(e_{i}-h_{i}-\dot{y}_{i}\right)-e_{i} h_{i}-e_{i} \dot{y}_{i} \\
= & b_{i} k_{i}^{2} \cos \left(k_{i}\right)\left(e_{i}^{2}-e_{i} h_{i}-e_{i} \dot{y}_{i}\right)-e_{i} h_{i}-e_{i} \dot{y}_{i} \\
& +e_{i}^{2}-e_{i}^{2} \\
= & {\left[b_{i} k_{i}^{2} \cos \left(k_{i}\right)+1\right]\left(e_{i}^{2}-e_{i} h_{i}-e_{i} \dot{y}_{i}\right)-e_{i}^{2} } \\
& \leq\left[b_{i} k_{i}^{2} \cos \left(k_{i}\right)+1\right]\left(e_{i}^{2}-e_{i} h_{i}-e_{i} \dot{y}_{i}\right)
\end{aligned}
$$

Then integrating the two sides of equation (11) and inequality (12) respectively to obtain

$$
\begin{gathered}
V_{i}(t)=\int_{0}^{t}\left[b_{i} k_{i}^{2} \cos \left(k_{i}\right)+1\right] \dot{k}(\tau) d \tau \\
+V_{i}(0)-\int_{0}^{t} e_{i}^{2}(\tau) d \tau \\
V_{i}(t) \leq \int_{0}^{t}\left[b_{i} k_{i}^{2} \cos \left(k_{i}\right)+1\right] \dot{k}(\tau) d \tau+V_{i}(0)
\end{gathered}
$$

According to Lemma $1, V_{i}(t), k_{i}(t), \int_{0}^{t}\left[b_{i} k_{i}^{2} \cos \left(k_{i}\right)+1\right] \dot{k}(\tau) d \tau$ are bounded on $[0, t)$. From (13) $e_{i}$ is square-integrable. Note that $e_{i}$ is bounded because of $V_{i}=\frac{1}{2} e_{i}^{2}$. Then it follows from (7) that $x_{i}$ and $z_{i}$ are also bounded. Furthermore, from (6) and (8) $h_{i}$ and $\tilde{h}_{i}$ are bounded. Thus, according to $\dot{e}_{i}=b_{i} \tilde{h}_{i}-h_{i}-\dot{y}_{i}$, one has that $\dot{e}_{i}$ is bounded. Hence, there holds $\lim _{t \rightarrow \infty} e_{i}(t)=0$ according to Lemma 2 .

Let $z=\left(z_{1}, z_{2}, \ldots, z_{N}\right)^{T}, e=\left(e_{1}, e_{2}, \ldots, e_{N}\right)^{T}, x=\left(x_{1}, x_{2}, \ldots, x_{N}\right)^{T}, y=\left(y_{1}, y_{2}, \ldots, y_{N}\right)^{T}$, then $(7)$ can be rewritten as the following vector form:

$$
e=x-z-y
$$

Define $\alpha=z+y$, then (15) can be written as $e=x-\alpha$. According to (6), (15) and $\dot{y}_{i}=$ 0 , it yields

$$
\dot{\alpha}=\dot{z}+\dot{y}=-L x=-L(e+\alpha)=-L \alpha-L e
$$

in this case, the solution of (16) can be written as:

$$
\alpha(t)=\exp ^{-L t} \alpha(0)-\int_{0}^{t} \exp ^{-L(t-\tau)} L e(\tau) d \tau
$$


where $\exp$ is index function. Since $\lim _{t \rightarrow \infty} e_{i}(t)=0$ has been obtained through the above analysis, when $t \rightarrow \infty$, (17) can be written as:

$$
\alpha(t)=\exp ^{-L t} \alpha_{0}, t \rightarrow \infty
$$

where $\alpha_{0}=\alpha(0)$.

Let $\delta=\left[\delta_{1}^{T}, \delta_{2}^{T}, \cdots, \delta_{n}^{T}\right]$ be the eigenvector matrix of the Laplacian matrix after orthonormalization, corresponding to eigenvalues $0=\lambda_{1}(L)<\lambda_{2}(L) \cdots \leq \lambda_{n}(L)$, and then let $\Lambda(L)=\operatorname{Diag}\left(\left[\lambda_{1}(L), \ldots, \lambda_{n}(L)\right]^{T}\right)$. Through spectral decomposition, (18) can be expressed as:

$$
\begin{gathered}
\alpha(t)=\exp ^{-\lambda_{1}(L) t}\left(\delta_{1}^{T} \alpha_{0}\right) \delta_{1}+\exp ^{-\lambda_{2}(L) t}\left(\delta_{2}^{T} \alpha_{0}\right) \delta_{2}+ \\
\cdots+\exp ^{-\lambda_{n}(L) t}\left(\delta_{n}^{T} \alpha_{0}\right) \delta_{n}
\end{gathered}
$$

Since all the eigenvalues of $L$ are greater than 0 except for the eigenvalue $\lambda_{1}(L)$, there holds

$$
\alpha(t) \rightarrow\left(\delta_{1}^{T} \alpha_{0}\right) \delta_{1}=\frac{I_{n}^{T} \alpha_{0}}{n} I_{n} \text {, as } t \rightarrow \infty
$$

it is obvious to obtain $\lim _{t \rightarrow \infty}\left(\alpha_{i}(t)-\alpha_{j}(t)\right)=0, \forall i, j=1, \ldots, n$. In addition, as $e=x-$ $\alpha$ and $\lim _{t \rightarrow \infty} e_{i}(t)=0$, we can get $\lim _{t \rightarrow \infty}\left(x_{i}(t)-x_{j}(t)\right)=0, \forall i, j=1, \ldots, n$. Given that the graph is undirected, one has:

$$
\sum_{i=1}^{n} \dot{z}_{i}(t)=\sum_{i=1}^{n} \sum_{j \in N_{i}} a_{i j}\left(x_{j}(t)-x_{i}(t)\right)=0
$$

Since $\sum_{i=1}^{n} z_{i}(0)=0$, it can be obtained from (20) that $\sum_{i=1}^{n} z_{i}(t)=0$ for all $t \geq 0$. Then from (15) one obtains:

$$
\sum_{i=1}^{n} e_{i}(t)=\sum_{i=1}^{n} x_{i}(t)-\sum_{i=1}^{n} z_{i}(t)-\sum_{i=1}^{n} y_{i}(t)
$$

Resulting from $\lim _{t \rightarrow \infty} e_{i}(t)=0$ and $\sum_{i=1}^{n} z_{i}(t)=0$ when $t \rightarrow \infty,(21)$ becomes:

$$
\sum_{i=1}^{n} x_{i}(t)=\sum_{i=1}^{n} y_{i}(t)
$$

In the above development one has obtained $\lim _{t \rightarrow \infty}\left(x_{i}(t)-x_{j}(t)\right)=0$, combing with (22) one can conclude that $\lim _{t \rightarrow \infty}\left(x_{i}(t)-1 / n \sum_{j=1}^{n} y_{j}(t)\right)=0, \forall i, j=1, \ldots, n$. This completes the proof.

Theorem 2. For an undirected and connected network graph, if the measurement target is dynamic and there holds $\sum_{i=1}^{n} z_{i}(0)=0$, then the cooperative estimation of multi-sensor system (5) can achieve average consensus under the adaptive estimation algorithm (8), that is, $\lim _{t \rightarrow \infty}\left(x_{i}(t)-1 / n \sum_{j=1}^{n} y_{j}(t)\right)=0, \forall i, j=1, \ldots, n$.

Proof: The proof development of getting $\lim _{t \rightarrow \infty} e_{i}(t)=0$ of Theorem 2 is same to that of Theorem 1, thus it is omitted here. Since the main difference between Theorem 2 and Theorem 1 is whether $\dot{y}_{i}(t)$ is 0 , according to (6), (15) and $\dot{y}_{i} \neq 0$, we can get:

$$
\dot{\alpha}=\dot{z}+\dot{y}=-L x+\dot{y}=-L \alpha-L e+\dot{y}
$$

Defining $\hat{\alpha}=Q \alpha$ and multiplying $Q$ on both sides of (23), it yields:

$$
\dot{\hat{\alpha}}=-Q L \alpha-Q L e+Q \dot{y}
$$


Given that $y_{i}(t)=C s(t)+D v_{i}(t)$, and $v_{i}(t)$ is a time-invariant constant value, for $\forall i=1, \ldots, n$, from $\dot{y}_{i}(t)=C \dot{s}(t)$ one has $\dot{y}(t)=\beta(t) \mathrm{I}_{n}$ with $\beta(t)=C \dot{s}(t)$. In addition, according to the properties in (10), we can get $L Q^{T} Q=L\left(\mathrm{E}_{n}-\frac{1}{n} \mathrm{I}_{n} \mathrm{I}_{n}^{T}\right)$ and $Q \dot{y}=0_{n-1}$, then (24) can be written as:

$$
\dot{\hat{\alpha}}=-Q L Q^{T} Q \alpha-Q L e=-Q L Q^{T} \hat{\alpha}-Q L e
$$

Since all the eigenvalues of $Q L Q^{T}$ have positive real parts, one can concludes that system (25) is an ISS system with input $Q L e$ and state $\hat{\alpha}$, since from $\lim _{t \rightarrow \infty} e_{i}(t)=0$ one has $\lim _{t \rightarrow \infty} \hat{\alpha}(t)=0_{n-1}$. According to $(10)$, we can get $\operatorname{rank}(Q)=\operatorname{rank}\left(Q Q^{T}\right)=n-1$, this shows that the kernel space of $Q$ is one-dimensional. Thus there holds $Q \alpha=0_{n-1}$ if and only if $\alpha=a \mathrm{I}_{n}, a \in \mathbb{R}$, which in turn implies $\lim _{t \rightarrow \infty}\left(\alpha_{i}(t)-\alpha_{j}(t)\right)=0, \forall i, j=1, \ldots, n$. In addition, in light of $e=x-\alpha$, and $\lim _{t \rightarrow \infty} e_{i}(t)=0$, one gets $\lim _{t \rightarrow \infty}\left(x_{i}(t)-x_{j}(t)\right)=$ $0, \forall i, j=1, \ldots, n$. The rest proof is the same as that of Theorem 1. Finally, when $\dot{y}_{i}(t) \neq 0$, $\lim _{t \rightarrow \infty}\left(x_{i}(t)-\frac{1}{n} \sum_{j=1}^{n} y_{j}(t)\right)=0, \forall i, j=1, \ldots, n$, can be obtained. This completes the proof.

\section{Simulation}

In order to demonstrate the effectiveness of the theoretical results of this paper, the numerical simulations are carried out by using Matlab. This section will simulate a network with four sensors. First, considering the case with a static target, then let $\boldsymbol{s}(\boldsymbol{t})=\mathbf{2}$. The constant interference of the sensors for the target during the measurement process are $\boldsymbol{v}_{\mathbf{1}}(\boldsymbol{t})=\mathbf{2}$, $v_{2}(t)=3, v_{3}(t)=4$ and $v_{3}(t)=5$. Supposing the output measurement matrix $C=1$ and the output interference matrix $D=1$, so the measured values of the four sensors are $y_{1}(t)=$ 4, $y_{2}(t)=5, y_{3}(t)=6$ and $y_{4}(t)=7$. Let $b_{1}=-1$ of the estimated model in the first sensor, and set the other sensors as normal, that is, $\boldsymbol{b}_{\mathbf{2}}=\boldsymbol{b}_{\mathbf{3}}=\boldsymbol{b}_{\mathbf{4}}=\mathbf{1}$. Set the initial state of the estimated model of the sensors as $\boldsymbol{x}(\mathbf{0})=[\mathbf{2}, \mathbf{4}, \mathbf{6}, \mathbf{8}]^{T}$, the initial state of the reference model as $\boldsymbol{Z}(\mathbf{0})=[\mathbf{0}, \mathbf{0}, \mathbf{0}, \mathbf{0}]^{T}$. Letting the communication network of the sensors be a loop diagram, then its adjacency matrix $\boldsymbol{\pi}$ is:

$$
\pi=\left(\begin{array}{llll}
0 & 1 & 1 & 0 \\
1 & 0 & 0 & 1 \\
1 & 0 & 0 & 1 \\
0 & 1 & 1 & 0
\end{array}\right)
$$

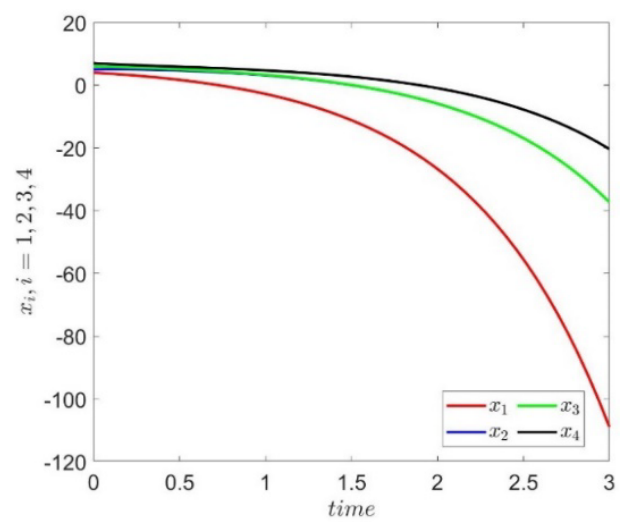

Fig. 1. The estimation of the static target using the estimation algorithm (3). 


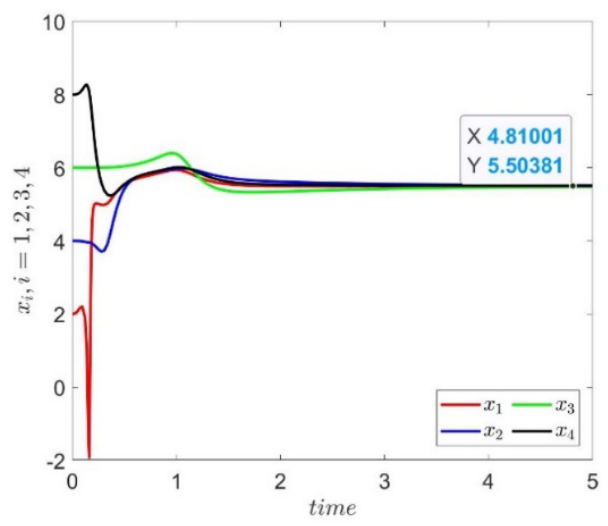

Fig. 2. The estimation of the static target using the estimation algorithm (8).

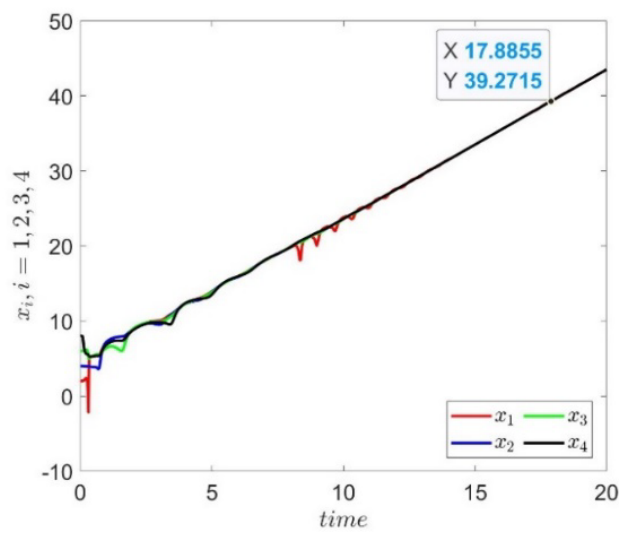

Fig. 3. The estimation of the moving target using the estimation algorithm (8).

Figure. 1 shows that for a static target, all estimation states will diverge when $b_{1}=-1$ by using the traditional estimation algorithm $\dot{x}_{i}(t)=-\sum_{j \in N_{i}} a_{i j}\left(x_{i}(t)-x_{j}(t)\right)$. After adopting the adaptive algorithm proposed in this paper, the estimation results are shown in figure.2, which indicates that the estimated states of four sensors can achieve consensus, particularly, the consensus state finally converges to the average value of all sensor measurement values.

When the target to be measured is dynamic, let $s(t)=2 t$, there still exists multiplicative interference $b_{1}=-1$ on the sensor 1 . As shown in figure.3, the adaptive estimation algorithm proposed in this paper can ensure the average consensus of the estimation results when the system is subjected to multiplicative interference.

\section{Conclusion}

In this paper, an adaptive algorithm with reference model is designed for the inaccurate estimation problems that may be encountered in the process of multi-sensor collaborative estimation, which can effectively overcome the influence of multiplicative interference factors on the system estimation results.

Note that there still exist some limitations of the method proposed in this paper. Firstly, this paper has made greater restrictions on the noise introduced in the target system to be measured and the multi-sensor measurement system, that is, it is required that they can only be time-invariant constants. Secondly, the multi-sensor measurement system is homogeneous 
in this article, but in practice, we prefer to be heterogeneous. Hence, in the future, we will further consider the introduction of Gaussian noise and heterogeneous multi-sensor measurement systems.

This work was supported by the Key Projects of Science and Technology Plan of Zhejiang Province under Grant 2021C01144.

\section{References}

1. Tilak S, Abu-Ghazaleh N B, Heinzelman W. A Taxonomy of Wireless Micro-sensor Network Models[J]. ACM SIGMOBILE Mobile Computing and Communications Review, 2002, 6(2):28-36.

2. Gungor V C, Lu B, Hancke G P. Opportunities and Challenges of Wireless Sensor Networks in Smart Grid[J]. IEEE Transactions on Industrial Electronics, 2010, 57(10):3557-3564.

3. Ge X, Han Q L, Zhang X M, et al. Distributed Event-triggered Estimation over Sensor Networks: A Survey[J]. IEEE Transactions on Cybernetics, 2020, 50(3):1306-1320.

4. Ding D, Han Q L, Wang Z, et al. A Survey on Model-based Distributed Control and Filtering for Industrial Cyber-Physical Systems[J]. IEEE Transactions on Industrial Informatics, 2019:2483-2499.

5. Reppa V, Papadopoulos P, Polycarpou M M, et al. A Distributed Architecture for HVAC Sensor Fault Detection and Isolation[J]. IEEE Transactions on Control Systems Technology, 2015, 23(4):1323-1337.

6. Diallo D, Diao S, Delpha C. Current Sensor Fault Estimation in The (d,q) Rotating Synchronous Frame. IECON 2016-42nd Annual Conference of the IEEE Industrial Electronics Society, 2016:6323-6328.

7. Ye X, Jiang J. Adaptive Nonlinear Design Without A Priori Knowledge of Control Directions[J]. IEEE Transactions on Automatic Control, 1998, 43(11):1617-1621.

8. Scardovi L, Arcak M, Sontag E D. Synchronization of Interconnected Systems With Applications to Biochemical Networks: An Input-Output Approach[J]. IEEE Transactions on Automatic Control, 2010, 55(6):1367-1379.

9. Min Y Y, Liu Y G. Barbalat Lemma and Its Application in Analysis of System Stability[J]. Journal of Shandong University(Engineering Science), 2007, 37(1): 51-55.

10. Doostmohammadian M, Meskin N. Sensor Fault Detection and Isolation via Networked Estimation: Full-rank Dynamical Systems[J]. IEEE Transactions on Control of Network Systems, 2020, PP(99):1-1.

11. Yu Y, Peng S, Li Q, et al. Distributed State Estimation for Heterogeneous Mobile Sensor Networks with Sensor Faults[C]. 2019 Chinese Control Conference (CCC), 2019.

12. Li H X, Wei W, et al. Fault Estimation in Finite Frequency Domain for Uncertain Networked Control Systems with Sensor Fault[C]. 2019 Chinese Control and Decision Conference, 2019.

13. Cao L, Li H, Dong G, et al. Event-triggered Control for Multiagent Systems with Sensor Faults and Input Saturation[J]. IEEE Transactions on Systems, Man, and Cybernetics: Systems, 2019, PP(99):1-12.

14. Lee Y D. Implementation of Greenhouse Environment Monitoring System Based on Wireless Sensor Networks[J]. Journal of the Korea Institute of Information and Communication Engineering, 2013, 17(11): 2686-2692. 
15. Zhao Z, Kuendig S, Carrera J, et al. Indoor Location for Smart Environments with Wireless Sensor and Actuator Networks[C]. 2017 IEEE 42nd Conference on Local Computer Networks (LCN), 2017.

16. Zhao X, Zhang X, Sun Z, et al. New Wireless Sensor Network Localization Algorithm for Outdoor Adventure[J]. IEEE Access, 2018, PP(99):1-1.

17. Lee, Huang C. Towards A General Wireless Sensor Network Platform for Outdoor Environment Monitoring[J]. 2012 IEEE Sensors, 2012:1-5.

18. Wang L, Zhao W, Yun L, et al. Sleep-supported and Cone-based Topology Control Method for Wireless Sensor Networks[C]. IEEE International Conference on Networking, 2008.

19. Liu S, D Tu, Zhang Y. Multiparameter Fire Detection Based on Wireless Sensor Network[C]. IEEE International Conference on Intelligent Computing and Intelligent Systems, 2009.

20. Jude M, Diniesh V C. DACC: Dynamic Agile Congestion Control Scheme for Effective Multiple Traffic Wireless Sensor Networks[C]. 2017 International Conference on Wireless Communications, Signal Processing and Networking (WiSPNET), 2017.

21. Xie X, Guo J, Zhang H, et al. Neural-network Based Structural Health Monitoring with Wireless Sensor Networks[C]. 2013 Ninth International Conference on Natural Computation (ICNC), 2013.

22. Chen B, Hu G, Zhang W A, et al. Distributed Mixed H2/H_infinity Fusion Estimation with Limited Communication Capacity[J]. IEEE Transactions on Automatic Control, 2015:805-810.

23. Xiao J J, Ribeiro A, Luo Z Q, et al. Distributed Compression-estimation Using Wireless Sensor Networks[J]. Signal Processing Magazine IEEE, 2006, 23(4):27-41.

24. Afsar M M, Mohammad H, Kh E Z. A Fault Tolerant Protocol for Wireless Sensor Networks[C]. Seventh International Conference on Mobile Ad-hoc \& Sensor Networks, 2011.

25. Bhuvaneswari P, Raj G V A, Balaji R, et al. Adaptive Traffic Signal Flow Control Using Wireless Sensor Networks[C]. 2012 Fourth International Conference on Computational Intelligence and Communication Networks (CICN), 2012. 